\title{
A Dynamic Environment to the Learning of Geometric Concepts by Visually Impaired People: A Brazilian Case Study
}

\author{
Lidiane Figueira ${ }^{1}$, Cabral Lima ${ }^{2, *}$, Antônio Borges ${ }^{3}$, Adriana Soares $^{4}$ \\ ${ }^{1}$ Corporative Education Center, Celso Lisboa University UCL, Rio de Janeiro, Brazil \\ ${ }^{2}$ Computer Science Department, Federal University of Rio de Janeiro UFRJ, Rio de Janeiro, Brazil \\ ${ }^{3}$ Electronic Computing Institute, Federal University of Rio de Janeiro UFRJ, Rio de Janeiro, Brazil \\ ${ }^{4}$ Psychology Department, Postgraduate Program, Salgado de Oliveira University UNIVERSO, Niterói, Brazil
}

\begin{abstract}
The learning of geometric concepts by Visually Impaired People (VIP) is a huge challenge. This paper presents a new dynamic computer-based environment for the learning of geometric concepts through adaptive technology. A case study on learning of geometric concepts in VIP classrooms using the proposed environment is detailed. Several experiments carried out with signed subjects (control group) and VIP subjects (experimental group) using the proposed method is also discussed. The results of this case study have shown that: $i$. the learning of geometric concepts by the VIP students was done through a peremptory and autonomous way; ii. the VIP students improved their ability to learn, retain and apply obtained concepts in other contexts; iii. the environment innovated the VIP geometry learning and increased their logical reasoning $i v$. the continuous use of the environment have enabled them to improve their spatial positioning and motions; $v$. the environment exhibited a superior performance than the classical geometry teaching in VIP classrooms. The main result of the experiments is that VIP students required (in average) only $20 \%$ of the time that was required in classical classes for solve correctly all proposed exercises.
\end{abstract}

Keywords Adaptive technology, Visually impaired people, Learning of geometric concepts, Computer-based environment

\section{Introduction}

The learning of geometric concepts by Visually Impaired People (VIP) is usually an intricate task due to its spatial characteristics that require understanding and manipulation of graphs, charts, lines, curves, solids etc. and, sometimes, visual conjectures and representations of spatial motions of an object. This intricacy may be attenuated by the use of devices and tools that allow the exploration of geometric concepts through the touching, but this does not eliminate the complexity of the learning of geometry by people with severe visual impairment or blindness. Nevertheless, the learning of geometric concepts is extremely important to VIP because geometry may improve other mental representations, sometimes very important when a VIP wants to move from a point to another in an actual environment.

This paper presents a Brazilian case study on VIP

* Corresponding author:

cabrallima@ufrj.br (Cabral Lima)

Published online at http://journal.sapub.org/computer

Copyright $\odot 2015$ Scientific \& Academic Publishing. All Rights Reserved learning of geometric concepts through a new dynamic computer-based environment (specially designed and implemented to these purposes). The case study experiments took place during three years in actual VIP classrooms and their analyses and main results are also discussed in this paper. The research presented in this paper has as the main assumption that the use of computer-based dynamic environments in VIP learning processes may help them to be more confident to learn, to retain and to apply new abstract concepts in contexts different from those where the concepts had been learnt. The analyses of this case study have been developed through a descriptive and interpretive approach under a fundamentally qualitative analysis of interpretative behavior. The choice of this approach is supported by some important theories such as those discussed in [1] and [2]. Two important results of the case study were: $i$. VIP students showed that they effectively were able to learn geometric concepts and to apply them in different scopes; ii. the environment exhibited a superior performance than the classical teaching of geometry in VIP classrooms.

In regard to the learning of geometric concepts, a computer-based environment is usually superior to paper 
and pencil. The huge possibilities to visualize an object (and its movements) dissociate the learner's intention of sketching from the one of drawing. The learner is leaded to know geometric properties before sketching an object on a screen. This reduces the false (and common) concept that the learning of geometric concepts is an empirical activity based on the manipulation of a ruler and compasses through trails and errors processes. In fact, the learning of geometric concepts using a computer-based environment generally releases the learner of implicit references associated to manipulations during the reasoning process to draw from a geometric problem enunciation, thanks to the mediation of knowledge supported by such environment.

In regard to Brazilian researches on learning having as target people with disabilities, [18] tried to improve cognitive gains of a child with special needs through a game on memorization of colors and sounds of virtual objects. The game uses algorithm of augmented reality; [19] tried to improve the cognitive gains of an autistic child through ludic activities using adaptive interfaces. In regard to researches on VIP learning, [20] discussed a system for controlling tactile geographical mockups and asserts that the use of speech synthesizer improves the perception of geometric connections; [21] proposed a system to assist a VIP to navigate on a diagram editor (the GEneric Diagram Editor, GEDE) in order to elaborate an UML diagram without help of someone else; [22] proposed a system to the learning of database entity-relationship diagrams (ERD), using a speech synthesizer, but without tutoring processes; [23] proposed a system to teach VIP typing on keyboards, based on the old techniques from typewriting classes, using a continuous feedback of a speech synthesizer.

The main contribution from [18] consists in allowing a therapist to plain a motion of a child who must memorize markers that had been put on the ground. The case study was with a 5 year old child with cerebral palsy. Unlike the research presented in this paper, [18] did not proposed any adjustment or actual facilitation for VIP: visual color recognition is a critical requirement to the progress of the proposed game. The research addressed to autistic children proposed by [19], differently from the research detailed in this paper, has as central contribution a thorough description of their software processes, do not having special attention to VIP learning processes. For instance, if an autistic child is a VIP he cannot use the system. Opposing the easiness of use of the environment proposed in this paper, the environment proposed by [20] use a lot of sensors, buttons, wires, electric contacts etc., which must be installed before using the environment, an additional difficulty for VIP. Moreover, the finger of the user is not tracked; only a sound is emitted when particular points are touched. [21] restricts the diagram development tool by allowing only the use of the keyboard and the screen reader, a stern deficiency for concrete manipulation of concepts as is supported by the environment proposed in this paper. Another handicap is that navigation must be done using predefined keystrokes that should be known in advance. In the environment proposed in this paper there is no requirement for prior knowledge and skills, as is the case of the system proposed by [22], in which a VIP student is required to already know data modeling since the diagram is developed from prior information provided by the user. The system requires also that a teacher fill a detailed specification of all problems to be proposed to the user. Differently from the environment proposed in this paper, in the system proposed by [23] there are no actual learning processes but a sort of training on typewriting. The system uses primal methods for the positioning of fingers and hands on the keyboard, using the ASDFG keys as a reference.

The proposed environment to the VIP learning of geometric concepts congregates "material" and "computational" components (such as a Webcam and learning algorithms) in a unique framework using simple and low cost adaptive technologies. The mathematical kernel of the proposed environment is responsible for calculating, from an image projection representation, what is the spatial point that gave rise to that projection. A key feature of this approach is based on the fact that the distances shown in perspective, regarding to objects placed farther, are represented distances that are smaller than the ones from the nearby objects. Besides exploring the interactive potential of the congregation of technologic artifacts, the proposed environment implements some strategies to the teaching of geometry. These strategies use mainly two approaches: the educational approach that is based on Piaget's constructivism and Papert's constructionist, and the technological approach that is based on pattern matching and image processing algorithms. The method uses a library of programs that have been specially designed and implemented in order to handle with all material and computational components.

The remainder of this paper is structured in four sections: section 2 examines the importance of the adaptive technology in VIP learning and its development in the Brazilian context; section 3 presents the material and methods of the proposed environment; section 4 details the experiments using the proposed environment; and section 5 shows the conclusions of this paper.

\section{VIP Learning}

\subsection{Computer-Based Adaptive Technology}

Although Adaptive Technology may be used as a synonym for Assistive Technology there is a substantial difference between them. Indeed, Assistive Technology, which was used for the first time in the IDEA 1990 (Individuals with Disabilities Education Act) [3], has a more general meaning because encloses adaptive and rehabilitative devices, i.e., any piece of equipment or product that may be used to help people with disabilities (enabling them to accomplish some complex tasks). Adaptive Technology is related to tools and systems that are specially designed for people with disabilities in order to help them to decrease their dependence on someone else in order to 
accomplish a task. Even if both terms are linked to the use of technology or technological artifacts, Adaptive Technology is addressed more commonly to electronic and computer-based technologies. An important use of Adaptive Technology is in learning processes, although this frequently requires some responsiveness (due to the transience and dynamic activations of several actions during a learning session) that is necessary to avoid violations of criteria of objectivity and pedagogical efficiency. Thus, the zetetic has been gradually shifted from the concern about if there exist (or not) beneficial aspects in the use of Adaptive Technology in learning processes, to the investigation on how Adaptive Technology can be properly and favorably applied in these processes.

\subsection{Adaptive Technology and VIP Learning}

The use of Adaptive Technology in VIP learning may still be considered modest. In the USA, for instance, only $59 \%$ of the $71 \%$ of VIP students (able to use this kind of technology) have a factual opportunity to do this [4]. Adaptive Technology has had an unimportant role in the whole education process due to two important factors: high cost, and small number of teachers who really bring significance to its use in actual classrooms. In Brazil the development of screen readers, Braille code printers, speech synthesizes, and mainly the Dosvox system [5], brought about the use of Adaptive Technology in VIP learning. Nevertheless, though these developments were a distinguished feature in the Brazilian VIP education, the learning of geometry by VIP was slightly targeted. Additionally, the technology was not sufficiently developed to offer tools able to enable a VIP to understand and manipulate geometric concepts. This restricted the VIP learning of geometry to be quite exclusively tactile, without significant involvement of Adaptive Technology.

\subsubsection{VIP Learning of Mathematics and Geometry}

Mathematics is usually renowned as a very difficult subject to be studied, very complex to be learnt and very intricate to be taught. Thus, the learning of mathematics by VIP is still considered as an enormous challenge. Indeed, unlike a text (which is linear and has unified standards, such as occurs in Braille code) a mathematic representation may have knotty structures that hinder a straight translation and understanding by a VIP. Complexity of expressions, multiple dimensions, nonlinearity, varied semantics and notations, spatial nature and numerous possibilities to express one idea, are usual characteristics of mathematics. These characteristics represent in fact an enormous defy for a VIP who wants to represent, interpret and manipulate a mathematic representation. The origin of chess may be a good example of this challenge. The story of the Grand Vizier Sissa Ben Dahir, who gave his new game to the Indian King Shihram, is intuitively easy to be understood by a sighted or a VIP, since they be able to elaborate a correct mental representation of a chessboard. Sissa wished to show to Shihram that a king needs - and must take good care of- all his subjects. Shihram was so content that he commanded that chess should be used in his kingdom due to its ability to train generals in the art of war and justice. Shihram ordered Sissa to ask him for a reward. Sissa solicited a grain of rice on the first square of the chessboard, two grains on the second, and so on (i.e., for each square the double of grains of the precedent square). This means 512 grains on the tenth square, 1048576 on the twentieth, and 9223372036854775808 $\left(9,22 \times 10^{18}\right)$ grains of rice on the last square. Considering $20 \mathrm{mg}$ as the mass of one grain of rice, Sissa's reward means 368.8 billion tons of rice. For instance, taking in account the 2014 world rice production, which was approximately 529 million tons [6], Sissa's reward would currently take around 697 years to be produced. A VIP can easily realize the semantics of this story, but to understand the mathematic meaning (and its symbolization) of this story is a bit more arduous.

$$
\begin{gathered}
S=1+q+q^{2}+q^{3}+\ldots+q^{n} \\
q S=q+q^{2}+q^{3}+q^{4}+\ldots+q^{n+1} \\
q S-S=q^{n+1}-1 \\
S=\left(q^{n+1}-1\right) /(q-1) \\
\sum_{i=0}^{i=63} 2^{i}
\end{gathered}
$$

Although very simple for sighted people, the mathematic representation of the amount of grains is a bit more complex for a VIP. For instance, the sum $S$ of the terms of a geometric progression with ratio $q \neq 1$ is done by the equation (1), which multiplied by $q$ results in (2). Diminishing (1) from (2) gives (3), which can be rewritten as (4). The final value of $S$ (5) is the summation representing Sissa's reward. Although this summation is very simple to be understood by sighted students it would require additional reasoning to VIP students.

$$
\frac{1+3}{2+5}
$$

The understanding, retention and manipulation of mathematic representations are the sine qua non condition to improve mathematic reasoning, a skill usually considered as arduous to inculcate in sighted students and much more complex in VIP students. The main sources of this complexity are:

a. mathematic representations are non-linear structures and even a small representation may be difficult to be understood by VIP students, even if it is said out loud as, for example, "one plus three divided by two plus five"(6). This expression could be understood as: "one plus, three divided by two, plus five": $1+(3 / 2)+5$ that is different from the first expression; 
$b$. the linearity of Braille code hinders a straight codification of mathematic structures;

c. correcting errors in Braille code is much more difficult than in texts. In fact, in Braille code this process requires a whole mental representation of a concept before writing it (if not, the writing must be restarted);

d. most teachers, largely in Brazil, receive little (if any) pedagogic guidance on how to select and apply methods dedicated to the VIP learning of mathematics.

In regard to the learning of geometry the most common environments are those for dynamic geometry. Conceptually, these environments allow elaboration of graphics and spatial manipulation of solids and curves. To accomplish this purpose these environments usually offer some tools such as virtual rulers, virtual compasses, graphic menus, and other resources routinely present in mathematical environments. A computer-based system allowing the exploration of spatial curves and solids, allied to tools that are able to offer concrete manipulations of geometric concepts, may give birth to a dynamic computer-based environment able to support the learning of geometry concepts and subsequent reifications of these concepts in actual environments.

Few environments for dynamic geometry learning have trigonometry as its essential goal [7], [8]. Although there are questionings on whether trigonometry belongs to geometry or to algebra, it seems quite intuitive to deal trigonometry as related to both. Indeed, geometry enables visual settings to the understanding of concepts of trigonometry, but once these concepts are retained they are normally incorporated to formulas, i.e., to the algebraic universe. In spite of trigonometry had born before algebra [9], its learning have got gain from this fusion, both for the conceptualizations addressed by the geometry (in benefice of algebra) and for the elimination of the overstated algebraic formalism when addressing to the geometry. Under this point of view, the resolution of triangles is the only subject genuinely belonging to trigonometry because everything else may bijectively be incorporated to algebra and to geometry.

\subsubsection{Adaptive Technology for VIP in a Brazilian Context}

Adaptive technology for VIP students is usually applied through two approaches: the static [10], [11], which basically uses the Braille code (sometimes an extended Braille code) for translating concepts, and the dynamic [12], [13], which has the audio as a fundamental tool for translating concepts.

The first development in Brazil of a computer-based system using adaptive technology was the drawing editor Desenvox [14] that used the speech synthesizer from Dosvox. The elaboration of a figure required to place elements on a planar panel (using selected coordinates) and subsequent displacements on the panel. This element placing involved basically the identification of the coordinates of the object while the displacements were a bit more complex because required to locate the desired element among the existent ones. Desenvox was discontinued because its use required that the student took a Braille code printer with him. In Brazil, the development of a new system using adaptive technology enabling a VIP to learn autonomously geometric concepts lasted until the development of the environment proposed in this paper.

In regard to computer-based systems of dynamic geometry that are more used in Brazil, it would be highlighted the GeoGebra [15], the Tabulae [16] and the Geometer's Sketchpad [17]. As its name shows, Geogebra merges algebra and geometry by linking geometric figures to their algebraic formulations. For instance, when an algebraic formula had been edited then a corresponding geometric figure is showed. A modification in the formula (or in related coordinates) modifies the figure. Tabulae has as main predicate the support for collaborative activities using mathematic concepts and objects. Another important virtue of Tabulae is the possibility of manipulating functions (polynomial, logarithmic, trigonometric etc.) whose changes can be observed in real time with the related geometric objects. The main potential from Geometer's Sketchpad is based on the learning of gradients, geometric transformations and arithmetic of integers, but it is also able to improve the learning of geometric concepts and figures through measurement and correlation properties. Another important virtue of Geometer's Sketchpad is its tools allowing manipulating properties of angles and polygons. Nevertheless, although these three systems are widely used in Brazil they are not focused on adaptive technologies and, unlike the environment proposed in this paper, they cannot be easily used by VIP.

\section{Material and Methods}

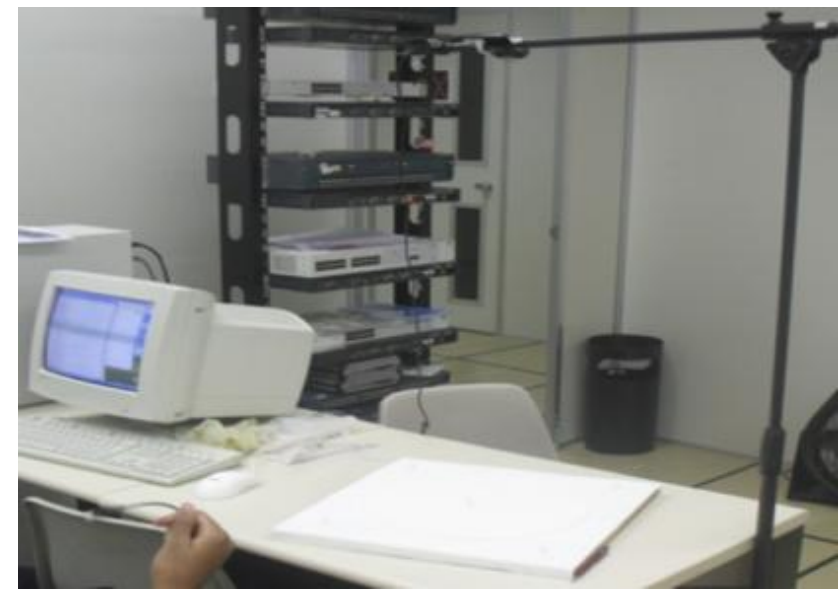

Figure 1. The components of the proposed environment

Besides the computer and the software itself, the proposed environment has as computational components: $i$. the Dosvox program (running as an interactive speech tool); $i$. handling routines; iii. image processing, pattern recognition and learning algorithms. The material components are a webcam, a pedestal and a board. The pedestal is very modest and may be replaced by any device allowing suspending orthogonally a webcam above the board (Figure 1). One 
important virtue of the environment is its very low cost and its easy configuration.

The central idea of the environment is to give to VIP students a real possibility of manipulate geometric concepts (and not just "read" or listen about them) and then to enable them to develop general abstract concepts from particular experiments. The use of adaptive technologies is crucial to enable them to consistently learn, retain and apply geometric concepts in an autonomous way.

\subsection{Development Method}

The developed method consisted of four phases: 1 . requirements definition; 2. exploratory study; 3 . student application; 4. contextual docent application. For the requirements definition a thorough in-loco study was done with VIP geometry classes, during one year, for 8th and 9th grades of the IBC [24], the most important organization for VIP assistance in Brazil. The main purpose of this first phase was to collect data and information required to the subsequent analysis of requisites. During this phase all the established processes that were used in IBC classes were carefully observed and noted, especially the processes used in geometry classes. The idea was to understand the actual educational scope and then to identify both the dynamic of the activation and the duration of the learning/teaching interactions. When this first phase was finishing, the first prototype of the software was developed in order to apply the experiments. The exploratory study was essentially linked to the software development life cycle of the environment, particularly its design, coding, test and deployment steps. The idea was to obtain additional subsidies to adapt the software interface to the sui generis needs of VIP users, taking in account the requirements issued from the first phase. This second phase demanded a huge data-testing process which required the implantation of several contexts to ensure the adequacy to the software feasibility and consistency. The student application used two groups of students: the first group (control group) had only sighted students and the second group (experimental group) had only VIP students. The contextual docent application was very important to the pragmatism of the environment, and took in account the required conformations issued from first and third phases analyses. Experiments involving VIP with concrete skills in mathematics, geometry and computer science (and who are available to effectively participate of long experiments) are rare because there are no many subjects congregating all these skills. Thus, this fourth phase was carried out through huge interactive sessions with a very smart computer scientist, M.Sc., teacher of geometry and blind.

The over-detailed executions of these four phases during three years has corroborate to confirm our hypothesis that the use of a dynamic environment in VIP learning can help them in processes of conception, retention and application of abstract concepts in other scopes. A further result of the execution of these four phases is that it was observed that despite the environment has been designed and implemented specifically for VIP, sighted users pointed out that its use facilitates the learning of geometry.

\subsection{The Environment}

The software of the environment has been designed and implemented under the software engineering rules in a such way that are appropriately covered all processes of design, implementation and testing (system engineering, requirements analysis, system design, architecture design, coding, validation and maintenance). The Py3K was used for implementing the main routines and the Pygame for controlling the webcam, computer graphics and sound effects. The environment uses image grabbing to further pattern matching. Filtering and image processing co-routines have been implemented to correct classic distortions of webcam images like cushion and barrel [25], [26]. The user's finger positioning image on the board is initially grabbed and the user is aware by a sound feedback informing him that the interaction had started. The development was modularized and global solutions were reified in one unique class in such way that all executable processes were consolidated and immediately available to be activated in running time. The main video module methods are: 1 ._init_: tracks and shows the webcam; 2. displayPropertyPage: returns the page properties; 3. display CaptureFilterProperty: responsible by the image filtering; 4. setResolution; 5. getBuffer: captures reading speeds; 6. getImage; 7. saveSnapshot: saves an image. The calibrating module is responsible to calibrate the webcam: the first user's finger image capture uses the $\mathrm{Z}$ standard calibration. The main methods of this module are: 1._init_: sets the webcam and grabs the first image of the board; 2. finger: tracks the user's finger; 3. calib: connects the four end points to run the calibrating process; 4. calc: calculates the geometric position of the current point; 5 . draw: captures the webcam reading speed; 6 . dTest: shows the calibrating points on the screen with the webcam current image. The option_calibrating module is responsible to allow the user to choose a manual or an automatic calibration. The manual calibration is required in the first use of the environment by a sighted person who must to adequate the board to the webcam visual field. The automatic calibration uses data of the first manual calibration to do a new calibration without a sighted intervention (enabling calibrations by VIP). The main methods of this module are: 1._init_: grabs the board image; 2. calibCam: uses data from the board in order to calibrate; 3. calibAuto: uses manual calibration data to do automatic calibrations.

A specific module calculates the inverse mapping projection (warping), i.e., an image from the board is in fact an object on a perspective view in order to simulate a visual sensation (as occurs in a photo). An important feature of this is that the distances vary with depth. Thus, the distances shown in perspective regarding to objects placed farther are represented with smaller distances than the distances from those closer. An important property (used in several 
processes) is that parallel lines from the visualized object converge all to a single point in the perspective. For example, an image obtained from a grid when viewed in perspective cannot simply be weighted as a result of a bilinear interpolation. Thus, calculating the inverse mapping means to discover, from a point of the image (projection), what is the point in space that gave rise to that projection. The solution to this problem requires conceptualizations and techniques of projective geometry that are computationally expensive and, if applied, could invalidate the actual use of the environment. To solve this problem an alternative solution was developed and has enabled the environment with an appropriate usability. This solution is an adaptation of the one proposed by [27], in which a camera recognizes points of a distorted polygon (captured image of a non-orthogonally focused rectangle) and turns them into $2 \mathrm{D}$ canonical coordinates. Although the [27] solution source code is a bit confidential, it had been possible to infer that the warping calculations came from a mathematic solution for texture mapping on surfaces [28]. In a general term, the solution developed to the proposed environment consists in solving a system of linear equations described through eight parameters ( $x, y$ coordinates of four points of the object plus the four similar points on the projection). This system of equations is represented by a transformation matrix that when multiplied by a column matrix (the projection coordinates) generates a new column matrix representing the projection. This was possible because a projected object was a canonical square object, i.e., the end points are represented by coordinates in $[0,1]$. Therefore, a transformation matrix can be properly generated, and when it is applied to a point $\mathrm{P}_{\mathrm{p}}=[\mathrm{x}, \mathrm{y}]$ of a projected image it generates a canonical point $P_{c}=[u, v]$. To obtain this point the adopted mathematic solution was based on the Heckbert's algorithm [28]. Thus, the points $[\mathrm{x}, \mathrm{y}]_{\mathrm{p}}$ will be precisely the points grabbed in the image and the points $[\mathrm{u}, \mathrm{v}]_{\mathrm{c}}$ will be the points to which they refer in a canonical board.

Finally, the main module is responsible to call all the other modules and to control all running processes in the environment. The main methods of this module are: 1 ._init_: load images; 2. exercPoint: functions for board point required in the phases; 3. exercLines: functions for board lines required in the phases; 4 . phase 1 to phaseN: routines of running processes in the phases; 5. challenge: logic for the challenge phase; 6 . quad1 to quad4: running processes of quadrants; 7. angProc: running processes for angles; 8. SinCosProc: running processes for sine and cosine; 9. update: running processes for positions and buttons; 10. draw: draw processes for images. All the running phases are permeated by computer processes that are activated by the module soundWin, which contains only one method with the same name: they are required by the speech synthesizer.

The learning processes of the environment were developed both from Piaget's constructivism (particularly in his studies on mental development that are started in the first years of life) and Papert's constructionist (especially regarding to the construction of knowledge from the interaction student-tool: a computer, for example). To Piaget the development of intelligence and cognitive ability is directly intertwined with the real-life experiences and the ability to understand the "logic-mathematic" discuss. This "learning-engine" is based on the ability to infer concepts that had not been necessarily taught. Corroborating to this idea, Papert argued that the insertion of a technologic tool in the educational scope of the student may result in his learning more significantly and autonomously. Papert alerted however that this constructionist approach occurs only if the inserted tool is adequate to the cycle "description-execution-reflection-debugging-description". Based on these assertions the proposed environment has been developed taking in account exploring and discovering processes, i.e., the user may interact with the objects from the environment and he may have the domain of the knowledge about how to properly use the retained concepts without obligatorily an instructional interference.

\section{Experiments}

The experiments were done using two different levels of exercises: Primary Level (PL) and Intermediate Level (IL). PL includes uncomplicated problems in terms of domain of geometric concepts. IL contains more complex problems, i.e., problems whose solutions require the domain of elaborated geometric concepts. Throughout PL the user can find six levels of geometric concepts and a seventh level that proposes a game that requires geometric location ability. This game was based on Simon game [29] in which the player is challenged to correctly memorize color and sound sequences. The subjacent idea of the seventh level is to instill the user to improve his skill in retaining and then applying concepts. Figure 2 illustrates activities of the fifth level where a student used the board (arrow 1) in order to draw a right triangle (helped by the environment through a sound) and then being aware of his score (that is also displayed with green dots for successes and black dots for errors: the other arrow). The main objective of the IL is to consolidate and expand the knowledge obtained in the PL throughout three steps: step 1: tests the user cognitive abilities on quadrants and positions; step 2: tests the user abilities to identify angles that had been generated in a random and contiguous way; step 3: tests how able the student is in retaining the geometric concepts involved in calculus of sine and cosine of angles.

The experiments revealed that the student application phase and the contextual docent application phase were especially corroborative to the hypothesis validation. In the student application phase a huge set of experiments was done in order to test the adequateness of the difficulty level to the PL. In the contextual docent application phase another huge set of experiments was similarly done in regard to the IL in which, besides all geometric concepts dealt in the PL experiments, spatial locations and trigonometry exercises were required. This occurred because during the third phase in IBC it had been verified that the students were not really taught about these concepts. 


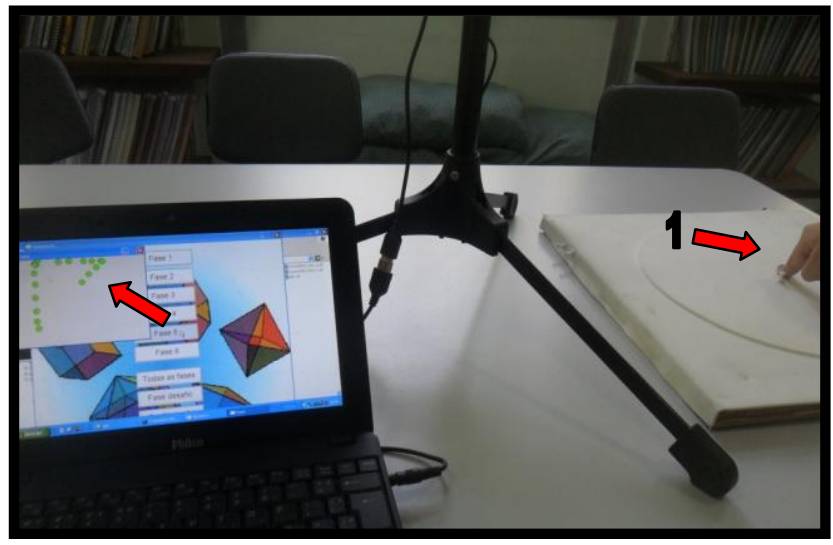

Figure 2. PL Fifth level: geometric concepts involved in a right triangle

In the experiments, four questionnaires (Q1 to $\mathrm{Q} 4)$ were presented to the subjects. In fact, Q1 (14 questions) and Q3 (17 questions) were applied in a preliminary stage of the experiments. These two questionnaires had as main objective to obtain socio-demographic data and academic traces, including information such as age, sex, VIP or not VIP, academic level, job, own computer, how many hours per day using computers, teacher of VIP, how difficult are geometry classes, technologies used in mathematic classes etc. After the subjects have answered Q1 and Q3 questionnaires they were put in contact with the environment to participate in actual experiments. After these experiments the questionnaires Q2 (7 questions) and Q4 (7 questions) were applied. The main goal of these two questionnaires was to obtain information about the environment itself, including information such as "AT equals VIP to sighted people in classical learning classes?", "what are the main environment behavior when dealing with VIP and when dealing with sighted people?", "how deep the environment helps VIP in spatial positionings?", "how deep the environment teaches geometric concepts?", "the environment really complements geometric classes?". Both questionnaires have closed questions based on Likert scale using five variables varying from "1. Strongly disagree" to "5. Completely agree". The questionnaires also provided an open space for subjects to express (without restrictions) their opinions, comments and questions about the experiments and the environment. These data were also analyzed using descriptive and interpretative approaches.

In the student application phase the experiments were done with two groups from 8th (11 students) and 9th (19 students) grades (VIP and sighted students) and 3 teachers of sighted students and 3 teachers of VIP students. In the control group all were sighted members and in the experimental groups there were 11 blind students, 1 student and 1 teacher with strong subnormal vision, and 2 sighted teachers (both having mainly observational tasks). The experiments occurred in the IBC and were monitored by the teachers. The student application phase used the following methodology: $i$. information about the proposal of the environment; $i i$. presentation of environment material tools; iii. application of Q1 and Q3 questionnaires; $i v$. webcam calibration; $v$. contextual explanation about how the environmental runs; vi. resolution of exercises (without interventions); vii. application of Q2 and Q4 questionnaires. It was observed that the blind students had difficulties in starting the environment, sometimes doing two times a same task and demanding around forty minutes to complete it.

The contextual docent application phase was done with a geometry teacher who is a blind computer scientist. The reason of having just one subject for this phase can be understood, as already explained, due to the fact that there are very few VIP (mainly in Brazil) with remarkable skill in mathematics, geometry, computer science and, mainly, that be a volunteer to participate in a research project involving a huge set of experimentations during three years. Of course, it is rare to find someone like Nicholas Saunderson, but the scientific skills of this subject have enormously improved all phases of the environment development. Some important information obtained during this experiment were: $i$. running precision analysis; $i i$. measuring answer speeds; $i i i$. verifying suitability and adequateness of exercises; $i v$. measuring degrees of abstraction, completeness of reasoning in problem solving; $v$. improving interface quality. When the students were considered ready to start using the environment, the teachers were required to answer the Q1 questionnaire. After that they were demanded to follow their students in the student application phase, and then they were required to answer the Q3 questionnaire (evaluating the use of the environment).

\subsection{Results and Discussion}

The main results obtained during the application of the seven levels are shown in Figure 3 (sighted subjects) and Figure 4 (VIP subjects).

To the hypothesis validation, besides the application of the descriptive-interpretive instruments, it were developed two processes: $i$. comparative study of student performances during problem-solving tasks; $i$. analysis of the significant data present in the commentaries when they were answering discursive questions (present in Q2 and Q4 questionnaires). Thus, for the comparative study, data representing performances were consolidated for the two groups, applying a scale with three rating levels (easy, intermediate, and difficult). For the data analysis, some discursive answers were randomly chosen and then a descriptive-interpretative analysis process was activated on such data. In addition, in order to improve and support some observed considerations, certain research information had been also quantified.

In the figures 3 and 4 the $x$ axis represents the levels (from L1 to L7) and the $y$ axis represents the quantity of subjects. From these figures it would be noted that the levels 1 and 2 (point locations) were successfully completed by all sighted students and by four blind students. The level 3 (horizontal and vertical lines: triangle's legs comprehension) was a little difficult to the blind students due perhaps the inexistence of a tactile support (like a string) for the user's finger (who was helped only by sound warnings). The level 4 (diagonal lines: hypotenuse comprehension) was successfully completed by 
four students in a first tentative; eight students demanded more than four tentative to obtain success. The students who gave up this level expressed that a diagonal requires a more train because it links to points differently positioned in the pad. The level 5 (right triangle comprehension) was difficult because of the handicap occurred in the phase 4. To understand the angles, legs and hypotenuse of a right triangle is effectively necessary to know about diagonals. The level 6 (perfect squares: sinus and cosines comprehension) and level 7 (the challenger game) have demonstrated an advancement in terms of usability of the environment, although the level 7 was a bit difficult to be accomplished even for sighted students. An important observation done by the subject of the contextual docent application phase was: "this environment is pioneer in the Brazilian VIP trigonometry learning because it has tactile and sound feedbacks supporting the learning of geometric concepts and the increasing of logical reasoning. The learning is obtained through a continuous and gradual growing of knowledge, from the simple point positioning to sinus and cosines of angles".

From the analysis in regard to the requirements definition it was inferred that: $i$. the environment must be really easy to use and must offer tactile signals helping the user; $i i$. the environment must give autonomy when the tasks are being carried out; iii. the environment must provide clear and understandable feedback to guide VIP users; $i v$. the environment must offer teaching material that may be used as a support for geometry class activities. The first prototype of the environment used these requirements analysis and, in a first moment, was informally experimented. Some enhancements of this prototype, initially got through trials and errors, were gradually implemented. Thus, three new prototypes were developed before all requirements definitions were wholly obeyed. The fourth prototype finally met all requirements definitions and become the first environment fully trustable for use in formal and actual experiments.

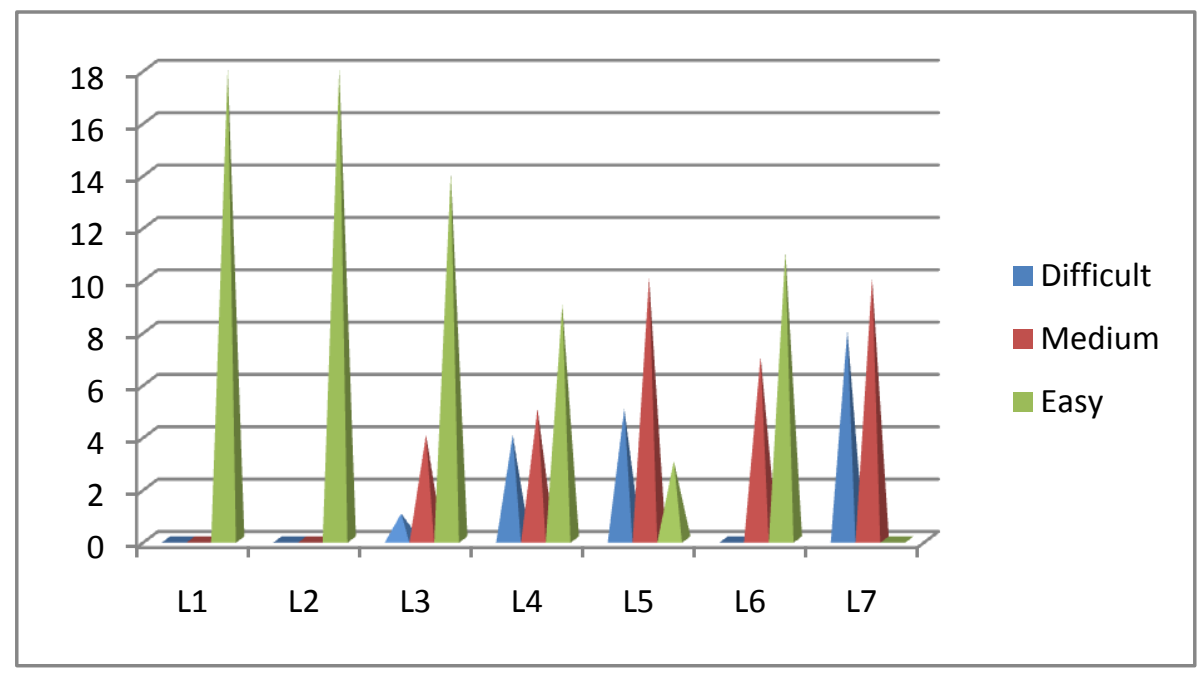

Figure 3. Sighted subjects - seven levels

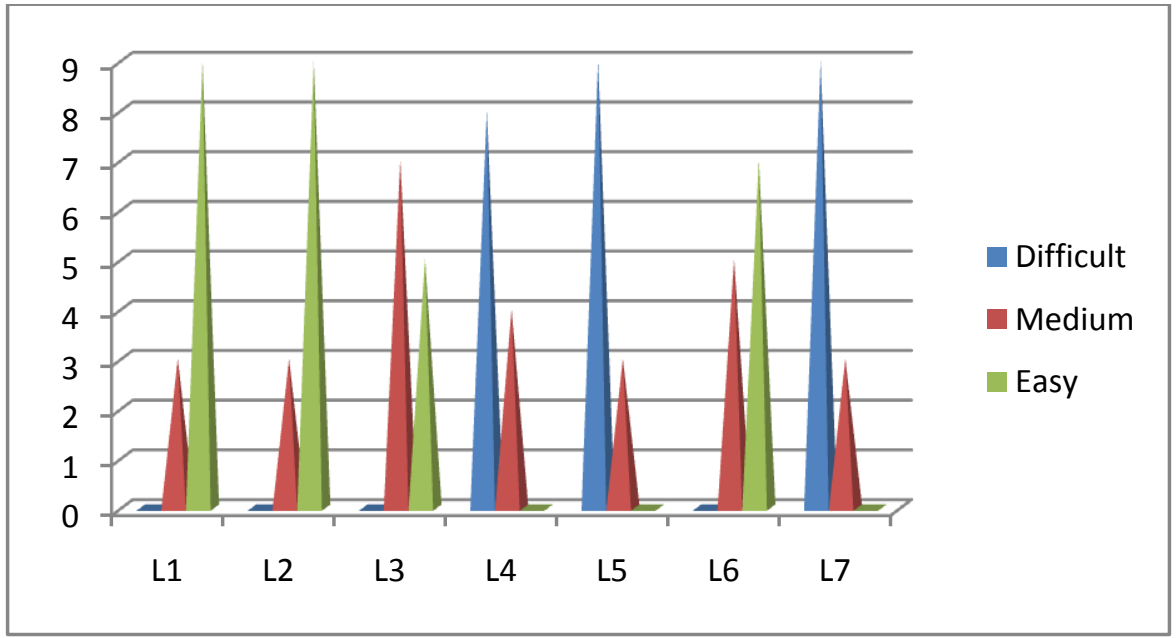

Figure 4. VIP subjects - seven levels 
In regard to the exploratory study it was necessary, in order to increase the environment versatility, to detect, filter and deal with the imaging scope, initially taking only points on the board. Further experiments showed that it would be really plausible to recognize the user's finger and calculate its spatial position on the board. The next step was consistently to adjust this to the speech synthesizer so that the user could understand what was being asked. Analyzing the data issued from these experiments it was concluded that the synchronism between the speech synthesizer and the running exercises was suitable, but the detection of points was not so good and demanded a supplementary refining process. Subsequent analysis of experiments with blindfolded sighted users revealed that the few instruction of that environment were also influencing the final result and inducing VIP to fail in solving problems. This revealed the necessity of new codes in the environment programming. This was extremely efficient for further experiments. Afterwards, a fourth battery of experiments was carried out with VIP who had knowledge on computer techniques. The analyses of these experiments revealed that the environment needed some functional enhancements in order to properly guide a VIP to use correctly the board. It was also considered that the time spent to answer a exercise should be optimized. Thus, concomitantly to the experiments all these adjustments were implemented, and then a novel and huge battery of experiments was done again. Although this cyclic way has demanded too much time and energy, the analyses in regard to this battery of experiments have proved be extremely promising and subsequently have corroborated to reach a steady and appropriate version of the environment.

In regard to the student application phase and contextual docent application phase the analyses of the results have corroborated to conclude that: $i$. there was a effective learning of geometric concepts; ii. students were able to solve exercises without extra interventions; iii. ludic activities increased the student logical reasoning; $i v$. the environment really helped the students (especially the VIP students) in the conception, retention and application of abstract concepts. Additionally, the descriptive analysis of the user reactions and, particularly, their contentment when they obtained success in concluding all phases (they often asked to keep using the environment) confirmed the achievement of the environment learning processes. The analyses regarding to the equation "time of acquiring conception/time of applying concepts" have clearly shown that the environment is more efficient than the classical teaching in the VIP classroom. For instance, using the environment, VIP students required (in average) 1/5 of the time that was required in classical classes for solve correctly the exercises of all phases.

The huge set of experiments carried out in the student application phase had as an important objective to verify the adequateness of the degree of difficulty level to the PL. Similarly, in the contextual docent application phase the applied experiments were to verify this adequateness in regard to the IL, in which, besides all geometric concepts dealt in PL experiments, spatial location and elaborated trigonometric concepts and exercises were addressed. Beyond the analyses described in section 4, additional descriptive-interpretative analyses were done using all instruments. An inspection on all user opinions (got from them immediately after they had used the environment) about suitability and usability of the environment, corroborates to validate the main research hypothesis, i.e., the use of computer-based dynamic environments in VIP learning processes may help them to be more confident to learn, to retain and to apply new abstract concepts in contexts different from those where the concepts had been learnt.

\section{Conclusions}

This paper presented a Brazilian case study on the learning of geometric concepts by Visually Impaired People (VIP) through a new dynamic computer-based environment. This case study lasted three years and was conducted with two groups of students (control group: sighted students; experimental group: visually impaired students) from 8th and 9th grades in actual classrooms. The main analysis of the case study was done through a descriptive and interpretive method under a fundamentally qualitative analysis of interpretative behavior. The experiments used different levels of knowledge, varying from Cartesian point localization to sinus and cosines comprehension and application.

The dynamic environment was developed using a four-phase method: requirements definition; exploratory study; student application; and contextual docent application. The requirements definition had as main objective to understand the education scope and to identify both the dynamic of the activation and the duration of the learning/teaching processes. The exploratory study was linked to the development of the software based on the main life cycle steps, i.e., designing, coding, testing and deployment. The student application was linked to the experiments with two groups of students, and the contextual docent application had as an important objective to adapt the environment to the required conformations issued from the first and third phases. The main advantages pointed out by the experiments with the proposed environment are: $i$. there is a peremptory and autonomous learning of geometric concepts; $i$. VIP students improved their logical reasoning and their skill in applying acquired concepts in other contexts; iii. the audible and tactile artifacts of the environment have shown be enough appropriate to allow VIP students to solve exercises without human helps; $i v$. some VIP students have related that the continuous use of the environment have enabled them to improve their spatial positioning and motions; $v$. the environment exhibited a superior performance than the VIP classical classes. 


\section{REFERENCES}

[1] J. W. Creswell. Qualitative Inquiry and Research Design. Sage Publications Inc, Second Edition. Thousand Oaks, CA, USA, 2007.

[2] J. W. Willis. Foundations of Qualitative Research: interpretative and critical approaches. Sage Publications Inc, Thousand Oaks, CA, USA, 2007.

[3] C. Kamei-Hannan, J. Howe, R. R. Herrera and J. N. Erin. Perceptions of teachers of students with visual impairments regarding assistive technology: a follow-up study to a university course. Journal of Visual Impairment \& Blindness. 106(10): 666-678, 2012.

[4] S. M. Kelly. Use of assistive technology by students with visual impairments: Findings from a national survey. Journal of Visual Impairment \& Blindness. 103(8): 470-480, 2009.

[5] J. A. Borges. From Braille to Dosvox - diferences in the live of the Brazilian blind people. Ph. D. thesis, Federal University of Rio de Janeiro, Rio de Janeiro, Brazil, 2008.

[6] S. Muthayya, J. D. Sugimoto, S. Montgomery and Glen F. Maberly. An overview of global rice production, supply, trade, and consumption Annals of the New York Academy of Sciences ISSN 0077-8923, pp. 7-14, Volume 1324, Issue 1.Setember, 2014.

[7] F. H. Ho and Y. H. Leong. Using dynamic geometry software in teaching geometry proof. In: Proceedings of the 5th East Asia Regional Conference on Mathematics Education, 2010.

[8] J. Zhang, X. Peng and M. Chen. Practice and thinking about teaching dynamic geometry course in normal university. Electronic Journal of Mathematics and Technology. 4(2): $1-15,2010$.

[9] N. J. Wildberger. Divine proportions: rational trigonometry to universal geometry. Sydney, Wild Egg, 2005.

[10] A. Edwards, H. McCartney and F. Fogarolo. Lambda: a multimodal approach to making mathematics accessible to blind students. Proceedings of the 8th International ACM SIGACCESS Conference on Computers and Accessibility. (1): 48-54, 2006.

[11] D. Crombie, R. Lenoir, N. McKenzie and A. Barker. Math2braille: opening access to mathematics. Proceedings of the International Conference on Computers Helping People with Special Needs. (1): 670-677, 2004.

[12] P. B. Atanley and A. I. Karshmer. Translating MathML into Nemeth Braille code. In: Proceedings of the International Conference on Computers Helping People with Special Needs. (1): 1175-1182, 2006.

[13] N. Soiffer. Mathplayer: web-based math accessibility. ASSETS3905, ACM Press, 2005.

[14] J. A. Borges, P. M. Barbosa, L. R. Jansen and S. B. Lyrio. Desenvox - computer tools to teach basic geometry and drawing for the visually disabled. 11th International Congress on Mathematical Education, Brazil, 2008.

[15] J. A. Edwards and K. Jones. Linking geometry and algebra with GeoGebra. Mathematics Teaching, 194, 28-30, 2006.

[16] Portal of Mathematical Collaboration. Available: http://tabulae.net/pcm/. Online, 2015.

[17] M. Satterfield. Geometer's Sketchpad: Single user package, V3. Mathematics, Teaching in the Middle School. 7(3): 184-194, 2001

[18] A. G. Corrêa, G. A. Assis, M. Nascimento and R. D. Lopes. GenVirtual: um jogo musical para reabilitação de indivíduos com necessidades especiais. Journal of Informatics and Education, 16(1): 09-17. Brazilian Computer Society. Brazil, 2008.

[19] F. R. Sousa, E. B. Costa and T. C. Castro. World-Tour: Software para suporte no ensino de crianças autistas. Proceedings of the XXIII Brazilian Congress on Informatics and Education, 2012.

[20] S. H. Ventorini. A experiência como fator determinante na representação espacial do deficiente visual. Msc. Thesis. UNESP Rio Claro, Brazil, 2007.

[21] L. G. Santos, A. M. Bandeira, L. T. Pansanato and D. B. Paiva. Recursos de acessibilidade para auxiliar a navegação de estudantes cegos em um editor de diagramas. Proceedings of the XXIII Brazilian Congress on Informatics and Education, 2012.

[22] R. L. Magalhães adn M. F. Neto. AprenDER: ferramenta de apoio à construção de diagrama entidade relacionamento para deficientes visuais. Proceedings of the XXI Brazilian Congress on Informatics and Education, 2010.

[23] [N. C. Albernaz, M. F. Elia ADN J. A. Borges. Digita-vox: curso de digitação com acessibilidade para deficientes visuais . Proceedings of the XXII Brazilian Congress on Informatics and Education, 2011.

[24] Instituto Benjamin Constant IBC. Available: http://www.ibc.gov.br/. Online, 2015.

[25] J. Mallon and P. F. Whelan. Precise radial undistortion of images. Proceedings of the 17 th International Conference on Pattern Recognition. (1): 18-21, 2004.

[26] T. H. Ngo and V. V. Asari. A pipelined architecture for real-time correction of barrel distortion in wide-angle camera images. IEEE Transactions on Circuits and Systems for Video Technology. 15(3): 436-444, 2005.

[27] J. C. Lee. Projector-based location discovery and tracking. Ph.D. Thesis. Carnegie Mellon University, 2008.

[28] P. S. Heckbert. Fundamentals of texture mapping and image warping, M.Sc. Thesis, Department of Electrical Engineering and Computer Science, University of California, Berkeley, 1989.

[29] S. Gould. Playing the game. GeriCon Books Ltda. ISBN 978-0956103475, 2010 